\title{
Exploring the 7p22.1 Chromosome as a Candidate Region for Autism
}

\author{
Nadia Bayou, ${ }^{1}$ Ahlem Belhadj, ${ }^{2}$ Hussein Daoud, ${ }^{3}$ Sylvain Briault, ${ }^{3}$ M. Bechir Helayem, ${ }^{2}$ \\ Habiba Chaabouni, ${ }^{1,4}$ and Ridha M'rad ${ }^{1,4}$ \\ ${ }^{1}$ Laboratoire de Génétique Humaine, Faculté de Médecine de Tunis, 15 rue Djebel Lakhdhar La Rabta, 1007 Tunis, Tunisia \\ ${ }^{2}$ Service de Pédopsychiatrie, Hôpital Razi, Cité des Orangers, 2010 La Manouba, Tunisia \\ ${ }^{3}$ Unité INSERM U619, Faculté de Médecine de Tours, 2bis, bd Tonnellé, 37000 Tours, France \\ ${ }^{4}$ Service des Maladies Congénitales et Héréditaires, Hôpital Charles Nicolle, Boulevard 9 Avril, 1007 Tunis, Tunisia \\ Correspondence should be addressed to Ridha M'rad, rmrad@yahoo.com
}

Received 3 December 2009; Revised 11 February 2010; Accepted 15 February 2010

Copyright (๑) 2010 Nadia Bayou et al. This is an open access article distributed under the Creative Commons Attribution License, which permits unrestricted use, distribution, and reproduction in any medium, provided the original work is properly cited.

\begin{abstract}
A high incidence of de novo chromosomal aberrations in a population of persons with autism suggests a causal relationship between certain chromosomal aberrations and the occurrence of autism. A previous study on a Tunisian boy carrying a $t(7 ; 16)$ translocation identified the 7p22.1 as a positional candidate region for autism on chromosome 7 . The characterization of the chromosomal breakpoints helped us to identify new candidate regions on chromosome 16p11.2 which contain no known genes and the other one on 7p22.1 containing a portion of genes (NP 976327.1, RBAK, Q6NUR6 also called RNF216L and MMD2). We proposed Q6NUR6 (RNF216L) as a candidate gene for autism due to its vicinity to the translocation breakpoint on the chromosome derivative 7. Q6NUR6 is predicted to be an E3ubiquitin-ligase. Quantitative PCR demonstrates that Q6NUR6 gene has an ubiquitous expression and that it is strongly expressed in fetal and adult brain. The Q6NUR6 expression is increased in the patient blood cells in comparison to controls. This is the first report of Q6NUR6 gene (E3 ubiquitin ligase TRIAD3 EC 6.3.2) increasing blood levels in a patient with autism. It's probably caused by a position effect involving this gene and modifying its expression.
\end{abstract}

A wide range of numerical and structural chromosome abnormalities including translocations, deletions, and duplications, visible in banding cytogenetic techniques, has been reported in autistic patients [1]. The high incidence of de novo chromosomal aberrations in a population of persons with autism suggests a causal relationship between certain chromosomal aberrations and the occurrence of autism [2].

We have studied a boy patient with autism and no dysmorphic features. Cytogenetic and molecular investigations revealed a de novo balanced translocation 46, XY, $t(7 ; 16)(p 22.1 ; p 11.2)$ in the patient. The karyotypes of the parents are normal. We mapped the breakpoints of the translocation on chromosomes 7 and 16 by fluorescence in situ hybridization (FISH). The characterization of the chromosomal breakpoints helped us to identify new candidate regions on chromosome $16 \mathrm{p} 11.2$ which contains no known genes and the other one on $7 \mathrm{p} 22.1$ containing a portion of genes (NP_976327.1, RBAK, Q6NUR6 also called RNF216L and MMD2) [3].
Metaphase spreads from the patient, her parents, and controls were obtained using standard protocols either from short-term blood lymphocyte cultures or from EBVtransformed lymphoblastoid cell cultures. Whole chromosome paints screen confirmed the presence of the $t(7 ; 16)$ translocation, showed that the chromosomal rearrangement implicates the chromosomes 7 and 16 only, and established that the translocation is reciprocal and apparently balanced (Figure 1(a)).

In order to finemap the breakpoint on $7 \mathrm{p} 22.1$, we performed FISH using BAC End clones from this chromosomal region as probes. BAC DNAs (RPCI-11 library) were biotinylated with biotin-11-dUTP (Sigma) by nick translation using the Bio Nick Labelling System (Invitrogen Life Technologies) according to the instructions of the manufacturers. Hybridization was performed using the standard procedures. After several rounds of hybridization, we identified one genomic BAC End clone (RP11-152I5) 


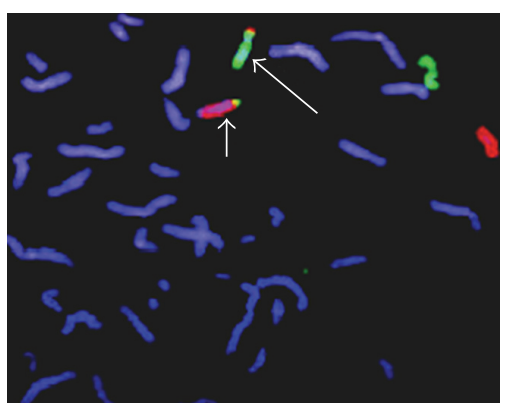

(a)
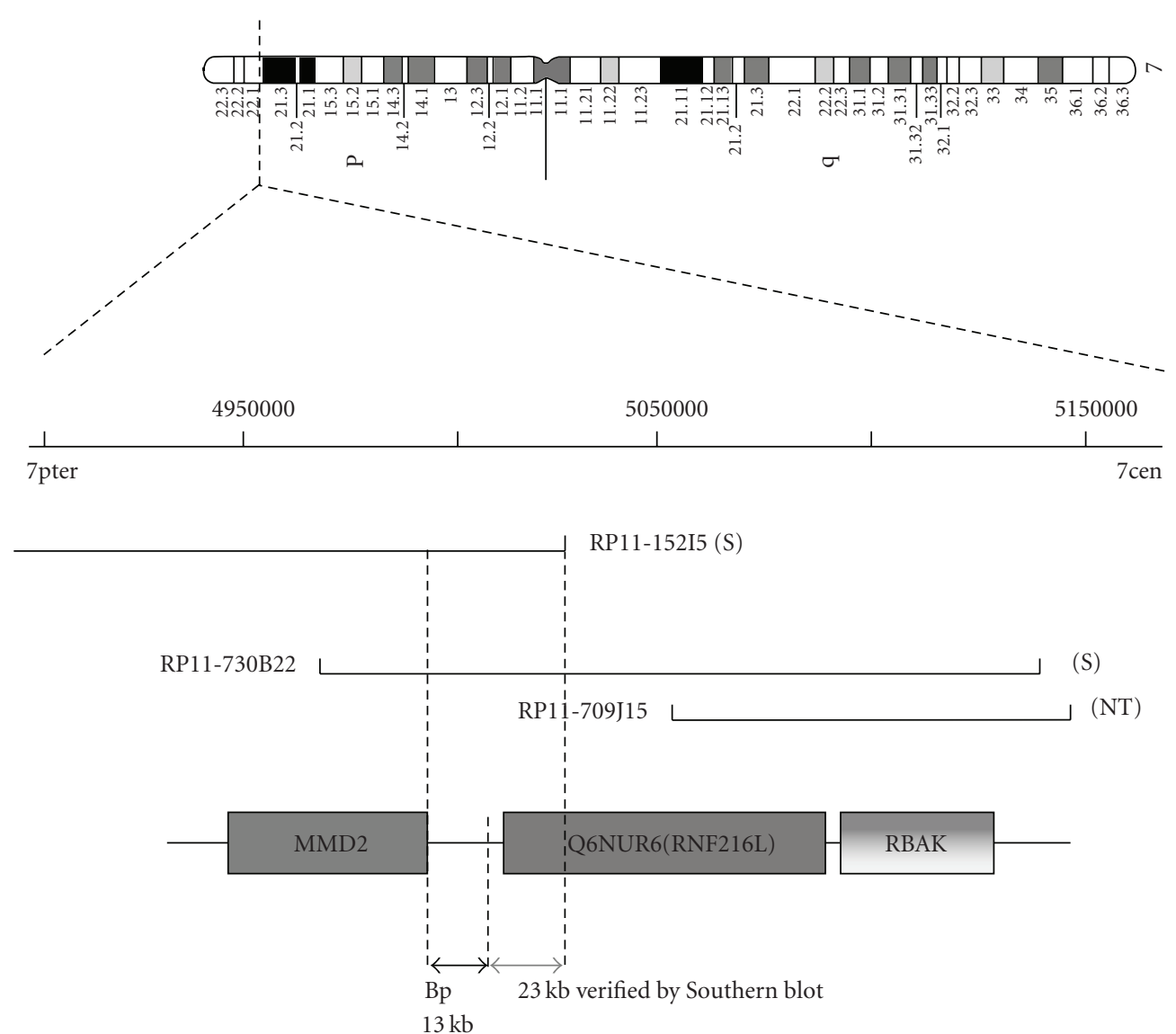

(b)

FIGURE 1: Physical mapping of the breakpoint on chromosome 7. (a) Dual color painting using directly FITC labelled (green) chromosome 7 paint and Rhodamine labelled (red) chromosome 16 paint. Both derivatives 7 and 16 (arrow) show clear evidence of a reciprocal exchange of chromatin from distal 7p and distal 16p, respectively. (b) Physical map of the genomic region 7p22.1 derived from the October 2009 version of the UCSC Genome Browser (http://genome.ucsc.edu/). The genomic clones covering the region flanking the translocation breakpoint are indicated by black lines ( $\mathrm{S}$ : spanning, NT: non-translocated). The breakpoint (Bp) region is represented by an arrow. The genomic location of candidate genes is represented by rectangles.

specific of band 7p22.1 and which is spanning the breakpoint on chromosome 7.

We analyzed 50 metaphases and noticed that the signal on chromosome derivative 16 was repeatedly larger than the signal of chromosome derivative 7 . These results suggest that the breakpoint on chromosome 7 is in the region corresponding to the centromeric half of BAC RP11-152I5 (Figure 1(b)).
Based on our results and according to the UCSC genome database (http://genome.ucsc.edu), we proposed Q6NUR6 (RNF216L) as a candidate gene for autism due to its vicinity to the translocation breakpoint on the chromosome derivative 7. The Q6NUR6 gene (GeneID: 441191) maps to chromosome 7p22.1 and contains 8 exons (only exons 4, 5 and 6 are coding), encompassing $24,183 \mathrm{~Kb}$ of genomic DNA. The gene transcribes an mRNA (BC068459) of 


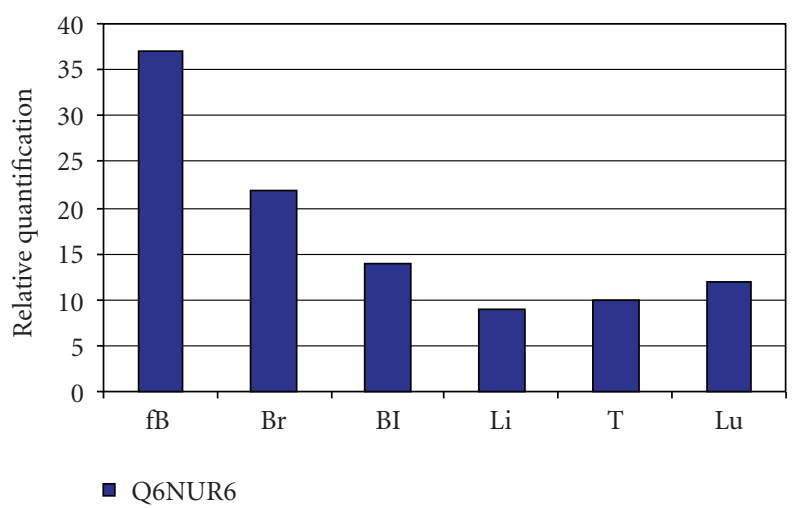

(a)

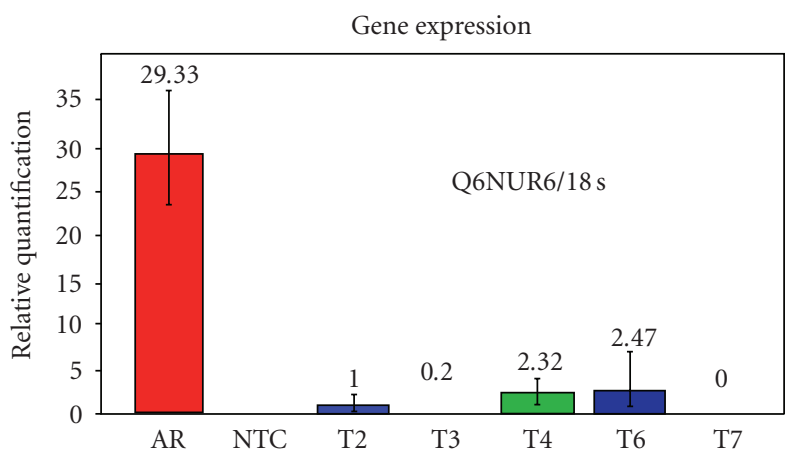

(b)

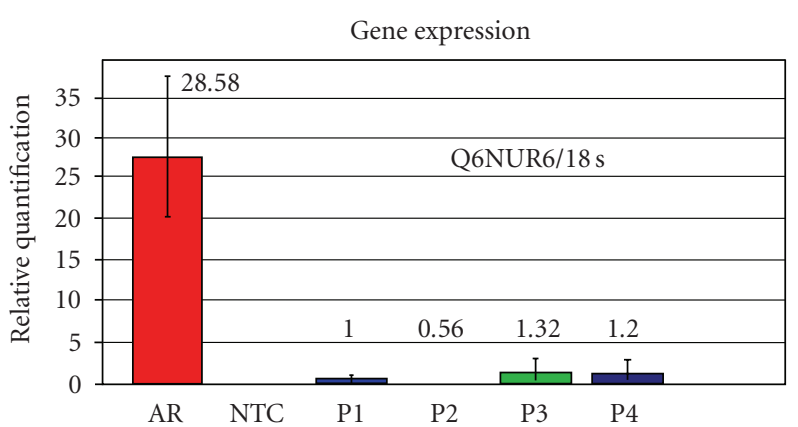

(c)

FIGURE 2: Quantitative expression of Q6NUR6 gene. (a) The expression pattern of the Q6NUR6 gene in various human tissues, fB: foetal brain; Br: brain; Bl: blood; Li: liver; T: testis; Lu: lung. (b, c) Quantitative Expression of Q6NUR6 gene in the patient and controls. QRTPCR was done on RNA extracted from blood cell lines. A significantly higher expression level was detected in the patient compared with normal control individuals (b) and with autistic patients without chromosomal anomalies (c). The expression normalization was done using the ribosomal 18S gene. (AR: Patient; NTC: No Template Control; T2, T3, T4, T6, T7: Healthy control individuals; P1, P2, P3, P4: autistic patients without chromosomal anomalies).

$2,427 \mathrm{~Kb}$ that encodes a 75 -amino-acid protein. The protein shows great homology with TRIAD3 gene. Its function is currently unknown but Q6NUR6 is predicted to be an E3 ubiquitin-ligase.

Approximately $23 \mathrm{~kb}$ of Q6NUR6 (including the promoter region) are located in the region corresponding to BAC RP11-152I5 (spanning the 7p22.1 breakpoint) (Figure 1(b)). To test whether this part of the gene was interrupted by the translocation breakpoint we performed Southern blot experiments to explore the genomic region containing the Q6NUR6 gene and its promoter on the patient's and a control DNA. All Southern blots gave normal results with restriction fragments at the expected sizes (data not shown), indicating that the Q6NUR6 gene or its promoter is not interrupted by the translocation breakpoint on chromosome 7.

Because Q6NUR6 gene is close to, but not interrupted by, the 7 p22.1 breakpoint, we wanted to determine if its expression is altered by the presence of the translocation breakpoint.

Total RNA was isolated and purified using the RNAgents Total RNA Isolation System (Promega) from peripheral white blood cells and EBV cell lines of the patient and controls. DNAse treatment was performed for 30 minutes with $1 \mathrm{mg}$ of RNA, $1 \mathrm{U}$ of RNAse-free DNAse I (Qiagen $\mathrm{GmbH}$, Germany). Total RNA was used in conjunction with AMV reverse transcriptase and random hexamers (Promega) to generate first strand cDNA. For the PCR step, $1 \mu \mathrm{g}$ of total RNA converted to cDNA was used in $25 \mu \mathrm{L}$ reactions with TaqMan Universal PCR Master Mix (Applied Biosystems, Foster City, CA, USA). For the Q6NUR6 gene the corresponding sequence-specific primers/probes assay mix (Hs00415445_m1) was used. Fluorescence detection was measured utilizing an ABI Prism 7500 platform (Applied Biosystems). After an initial step at $50^{\circ} \mathrm{C}$ for 2 minutes, there was an activation step at $95^{\circ} \mathrm{C}$ for 10 minutes. Thermal cycling proceeded with 50 cycles at $95^{\circ} \mathrm{C}$ for 15 seconds and 1 minute at $60^{\circ} \mathrm{C}$. Normalization to the ribosomal18s and to the beta-2-microglobulin (B2M) control genes was performed to account for variability in the initial concentration and quality of total RNA and in the conversion efficiency of the reverse transcription reaction.

All PCR reactions were performed in triplicate and changes in gene expression between the control (or calibrator) and treated samples after normalisation to the gene reference was calculated using the comparative threshold cycle $\left(C_{T}\right)$ method where relative amount is equal to $2^{-\Delta \Delta C T}$, 
and where $\Delta \Delta C_{T}$ is the $\Delta C_{T}$ of the target gene (threshold cycle test gene-threshold cycle endogenous control) minus the $\Delta C_{T}$ of the calibrator sample (threshold cycle calibrator gene-threshold cycle endogenous control).

Quantitative PCR demonstrates that Q6NUR6 gene has an ubiquitous expression and that it is strongly expressed in foetal and adult brain (Figure 2(a)). The Q6NUR6 expression is increased in the patient blood cells in comparison to controls (the control RNA originates from individuals without disease phenotype or chromosomal aberration). As shown in Figure 2(b), Q6NUR6 is overexpressed in the patient rather than in healthy control blood cells. The relative quantification of Q6NUR6 gene expression was established to 29.33 .

The average of the expression on normal control individuals was calculated as 0.9687 excluding a possible duplication of this gene. The threshold for overexpression was established as about 30-fold the value for the normal controls in EBV-cell lines.

To determine if this increase is caused by the translocation, we quantified the expression of Q6NUR6 in the patient's cell line and in four autistic patients without chromosomal aberrations (Figure 2(c)). The overexpression is the same that seen previously. This result means that the variation of Q6NUR6 gene expression seen in the patient cell line is probably caused by the translocation.

Quantitative RT-PCR was also used to verify if the remaining chromosome 7 candidate genes were differentially expressed in the patient and the control cases. NP_976327.1 gene was not expressed in blood and fibroblasts. RBAK and MMD2 genes were expressed at the same level in patient and controls (data available upon request).

The Q6NUR6 gene has been localized to $7 \mathrm{p} 22.1$ by the Mammalian Gene Collection [4]. This gene has been described as a pseudogene in Gene at NCBI database (www.ncbi.nlm.nih.gov/). However, it has been considered to be a protein coding gene by Ensembl Genome Browser (http://www.ensembl.org/). The Bgee database (http://bgee.unil.ch/) demonstrates that Q6NUR6 gene is expressed in a variety oh human tissues (like brain and hippocampus) at all developmental stages.

Our data demonstrate the ubiquitous expression of this gene and its overexpression in an autistic patient carrying a $\mathrm{t}(7 ; 16)(22.1 ; \mathrm{p} 11.2)$ reciprocal translocation.

Chromosomal aberrations, when they do not interrupt a gene, sometime lead to the modification of the expression of a gene due to the alteration of its environment, the so-called "position effect." The rearrangement may juxtapose the gene with an enhancer element from another gene leading to inappropriate gene expression [5]. This is the first report of Q6NUR6 gene (E3 ubiquitin ligase TRIAD3 EC_6.3.2) increasing blood levels in a patient with autism probably caused by a position effect involving this gene and modifying its expression.

Q6NUR6 is predicted as an E3 ubiquitin ligase from protein sequence homology with the TRIAD3 protein. Abnormalities in other E3 ubiquitin ligases have been described earlier in number of psychiatric diseases [6]. Mutations in E3 ligases were identified as causative for
Angelman syndrome [MIM:105830] (UBE3A gene) and recessive juvenile Parkinson disease [MIM : 600116] (PARK2 gene) [7].

In conclusion, all the data collected during the course of this study suggest that the Q6NUR6 gene may be implicated in the autistic phenotype of the reported patient, and that this phenotype is possibly caused by the presence of the translocation. The exact functions of Q6NUR6 gene in mammalian cells are still unclear and final genetic evidence linking this gene to autism will be obtained by the detection of mutations in this gene in other patients with autism, or the association of polymorphisms in this candidate gene with autism.

\section{References}

[1] J. A. S. Vorstman, W. G. Staal, E. van Daalen, H. Van Engeland, P. F. R. Hochstenbach, and L. Franke, "Identification of novel autism candidate regions through analysis of reported cytogenetic abnormalities associated with autism," Molecular Psychiatry, vol. 11, no. 1, pp. 18-28, 2006.

[2] B. J. O'Roak and M. W. State, "Autism genetics: strategies, challenges, and opportunities," Autism Research, vol. 1, no. 1, pp. 4-17, 2008.

[3] N. Bayou, R. M'rad, A. Belhaj, et al., "De novo balanced translocation $\mathrm{t}(7 ; 16)(\mathrm{p} 22.1 ; \mathrm{p} 11.2)$ associated with autistic disorder," Journal of Biomedicine and Biotechnology, vol. 2008, no. 1, Article ID 231904, 2008.

[4] D. S. Gerhard, "The status, quality, and expansion of the NIH full-length cDNA project: the Mammalian Gene Collection (MGC)," Genome Research B, vol. 14, no. 10, pp. 2121-2127, 2004.

[5] D.-J. Kleinjan and V. van Heyningen, "Position effect in human genetic disease," Human Molecular Genetics, vol. 7, no. 10, pp. 1611-1618, 1998.

[6] C. A. Bousman, G. Chana, S. J. Glatt, et al., "Preliminary evidence of ubiquitin proteasome system dysregulation in schizophrenia and bipolar disorder: convergent pathway analysis findings from two independent samples," American Journal of Medical Genetics, Part B, vol. 153, no. 2, pp. 494-502, 2009.

[7] I. Marin, J. I. Lucas, A.-C. Gradilla, and A. Ferrus, "Parkin and relatives: the RBR family of ubiquitin ligases," Physiological Genomics, vol. 17, no. 3, pp. 253-263, 2004. 

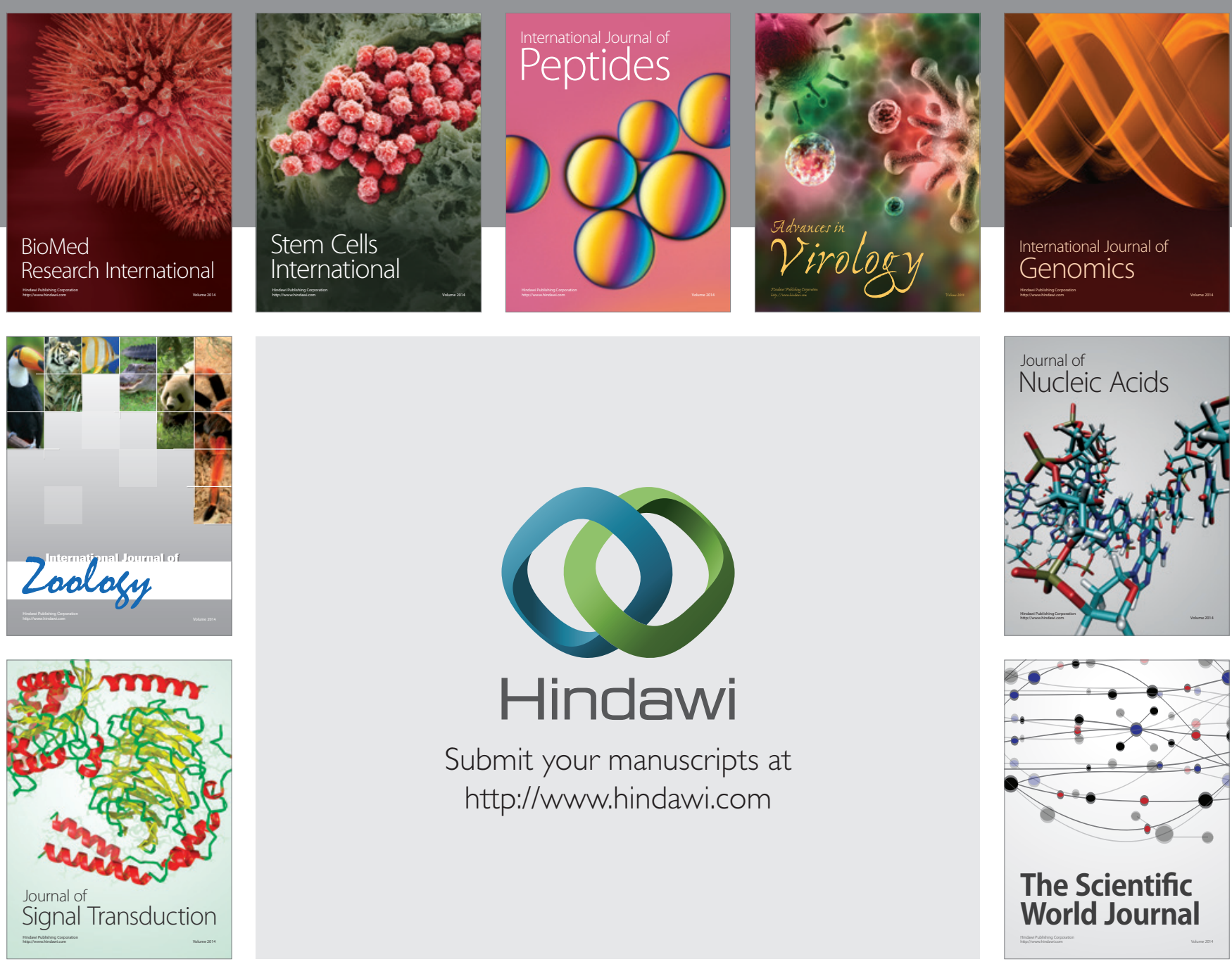

Submit your manuscripts at

http://www.hindawi.com
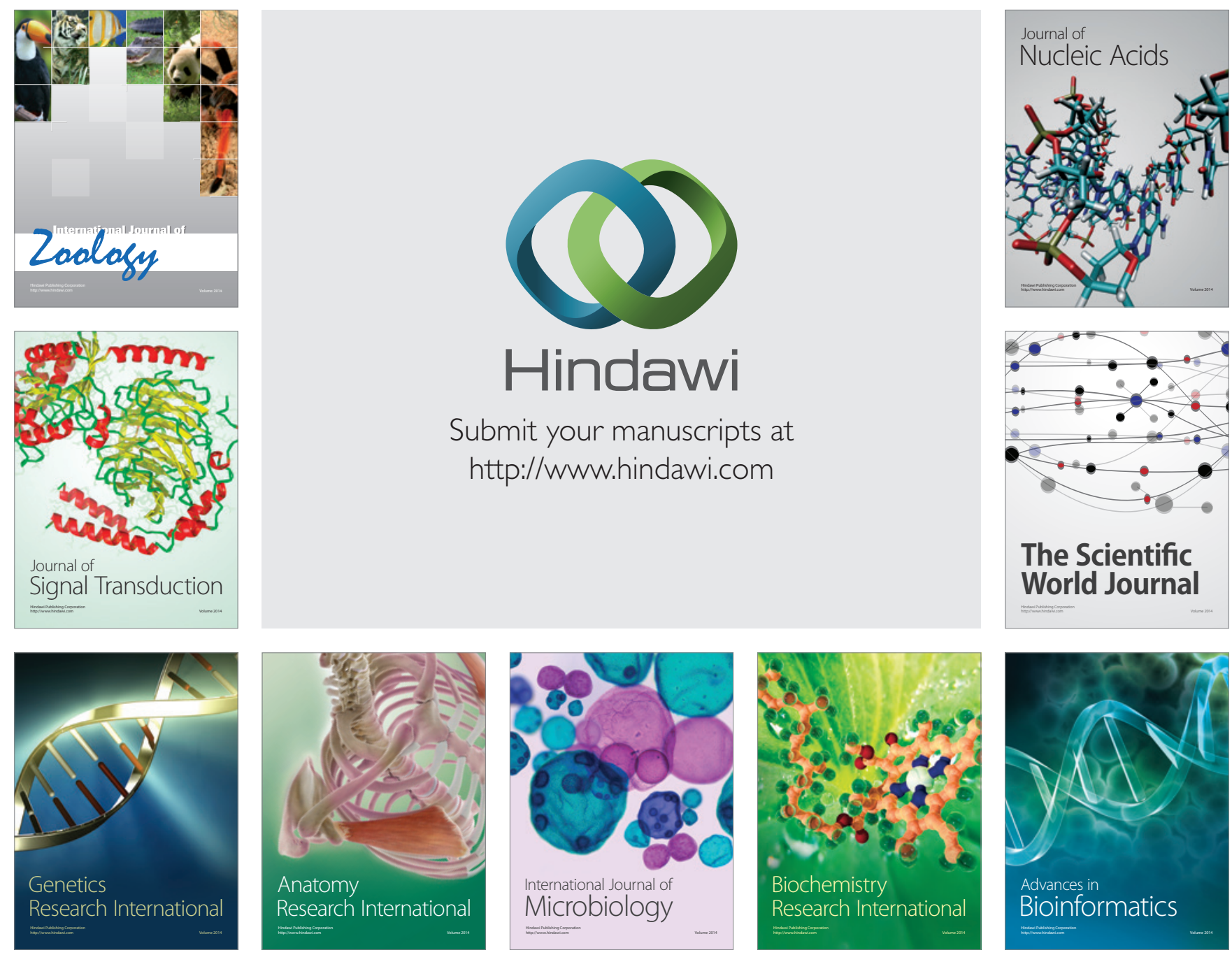

The Scientific World Journal
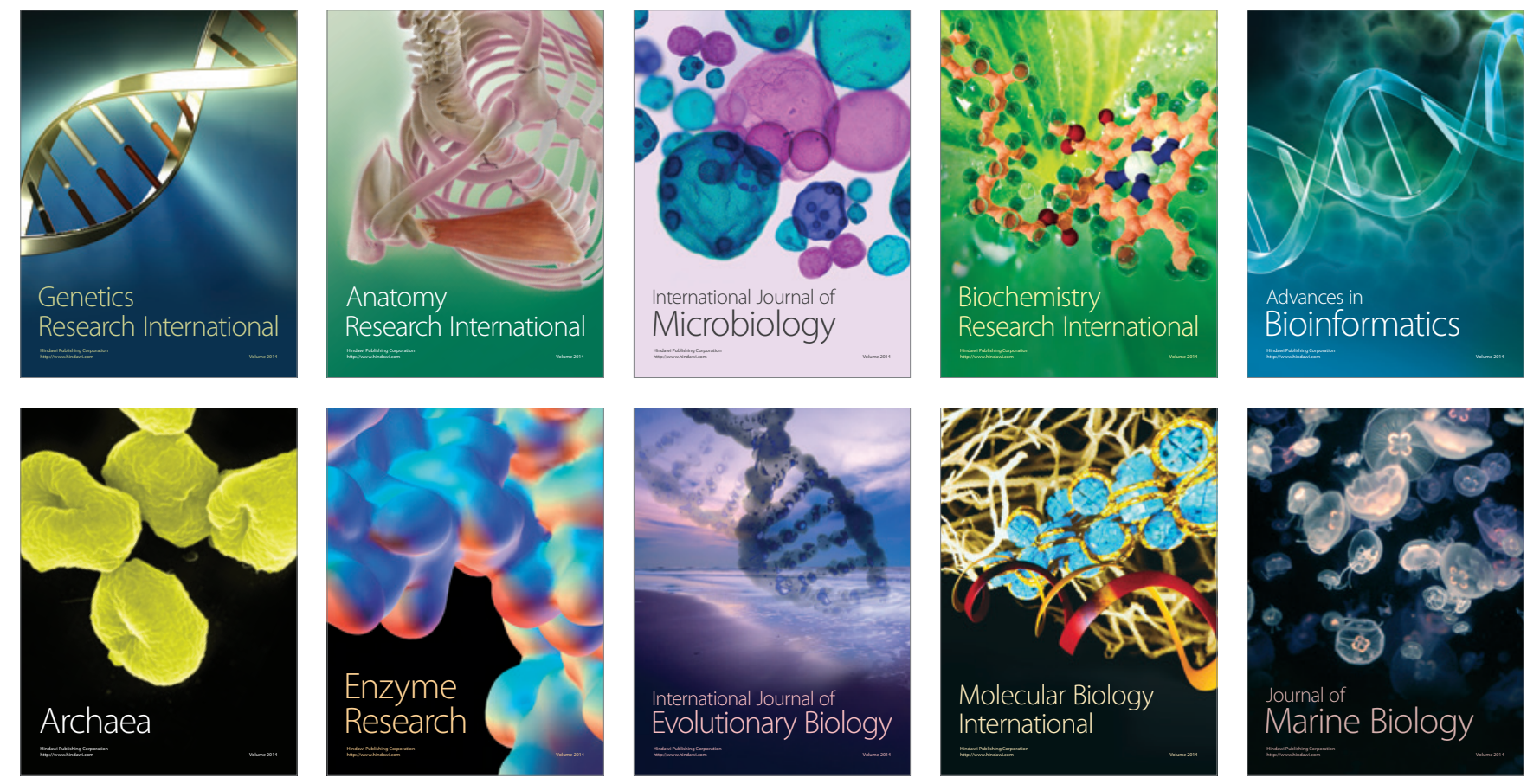